\title{
Comparative study of papanicolaou smear and colposcopy in the evaluation of cervical lesions
}

\section{Priyanka Mohan, Lakshmidevi M*, Shreedhar Venkatesh}

\begin{abstract}
Department of Obstetrics and Gynecology, Vydehi Institute of Medical Sciences and Research Centre, Bangalore,
\end{abstract} Karnataka, India

Received: 02 April 2019

Accepted: 06 May 2019

\section{*Correspondence:}

Dr. Lakshmidevi M.,

E-mail: dr_lakshmi_m1982@yahoo.co.in

Copyright: (c) the author(s), publisher and licensee Medip Academy. This is an open-access article distributed under the terms of the Creative Commons Attribution Non-Commercial License, which permits unrestricted non-commercial use, distribution, and reproduction in any medium, provided the original work is properly cited.

\begin{abstract}
Background: Cervical cancer is the third most common type of cancer among females. Study aims to critically evaluate the sensitivity and specificity of colposcopy versus papanicolaou (Pap) smear in the early detection of dysplasias. Its secondary objective to correlate the findings in the evaluation of unhealthy cervix by cytology, colposcopy and colposcopy guided biopsy.

Methods: This was a tertiary care teaching hospital based, prospective, cross sectional study done in Department of Obstetrics and Gynaecology, at Vydehi Institute of Medical Sciences and Research Centre, Bangalore, conducted on 200 women attending Gynaecology OPD.

Results: PAP smear was taken for all 200 patients. $73 \%$ of smear was found to be normal, $11 \%$ showed inflammatory atypia, 9\% showed low grade squamous intraepithelial lesion (LSIL), 3.5\% showed atypical squamous cells of undetermined significance (ASCUS) and 3.5\% showed High Grade Squamous Intraepithelial Lesion (HSIL). Among the 200 cases studied, $38 \%$ (76/200) were diagnosed as colposcopically abnormal. Among the abnormal cases, AW areas were diagnosed in $4 \%$. Punctate pattern of vessels was seen in 5\% of women. Normal findings was present in $62 \%$, Erosion cervix in $6 \%$, inflammatory changes were seen in $6 \%$ and polyps were diagnosed in $7.5 \%$, leucoplakia was found in $2 \%$ and unsatisfactory colposcopy finding was seen in $4 \%$ and underwent endocervical curettage. 32 cases out of 200 women were positive on Pap smear. 66 out of 200 women were positive on Biopsy. Pap smear was positive in 22 out of 66 biopsy proven positive cases.

Conclusions: The commonest presenting complaint was vaginal discharge $(182 / 200 ; 91 \%$ of the patients. the PAP smear is found to have sensitivity of $33.33 \%$ and specificity of $92.54 \%$. colposcopy is found to have sensitivity of $81.82 \%$ and specificity of $82.84 \%$.
\end{abstract}

Keywords: Colposcopy, Papanicolaou smear, Sensitivity and specificity

\section{INTRODUCTION}

According to the World Health Organization (WHO), cervical cancer is the third most common type of cancer among females, and was responsible for over 250,000 deaths in 2005. Approximately $80 \%$ of these deaths occurred in developing countries. Without urgent intervention, deaths due to cervical cancer are projected to rise by almost $25 \%$ over the next 10 years.

Early death due to cervical cancer and years affected by disease-related disability contributed to the loss of 6.4 million disability-adjusted life years (DALYs) globally in $2010 .{ }^{1}$ 
The World Health Organization global action plan on noncommunicable diseases describes screening with VIA as a tool, both highly cost-effective (i.e., it costs less than the per capita gross domestic product to avert one DALY) and feasible to implement in settings with constrained health systems. ${ }^{2}$ There are promising results from large trials suggesting that VIA can reduce cervical cancer incidence by $25 \%-30 \%$. $^{3,4}$ Although screening with HPV DNA testing is more expensive than with VIA, a study by Goldie and colleagues in five Low or Middle Income Countries (LMICs) found that HPV DNA screening is very cost-effective, and a single test at age 35 years reduces lifetime cancer risk by $25 \%-36 \% .5,6$ Integrating screening into primary care services for women should increase the likelihood that precancer is detected, as is seen in high-income countries, where effective screening averts progression to cervical cancer

The present study is an endeavor to perform a comparative study of performance of colposcopy and Pap smear for mass screening of premalignant and malignant lesions of the cervix. Study aims to critically evaluate the sensitivity and specificity of Colposcopy versus Pap smear in the early detection of dysplasias. Its secondary objective being, to correlate the findings in the evaluation of unhealthy cervix by cytology, colposcopy and colposcopy guided biopsy.

Objectives of the study were to critically evaluate the sensitivity and specificity of Colposcopy versus Pap smear in the early detection of dysplasias. To correlate the findings in the evaluation of unhealthy cervix by cytology, colposcopy and colposcopy guided biopsy.

\section{METHODS}

This was a tertiary care teaching hospital based, prospective, cross sectional study done in Department of Obstetrics and Gynaecology, at Vydehi Institute of Medical Sciences and Research Centre, Bangalore, India. The study was conducted on 200 women attending Gynaecology OPD. As the primary variables like sensitivity and specificity of PAP smear and unaided visual inspection of the cervix after an application of acetic acid (VIA) and Lugol's iodine (VILI) are expected to have effect size of between $20-60 \%$. $^{6}$

Author selected the confidence interval of 10 and confidence level of $95 \%$ for a population size of 25000 patients while deciding the sample size. The sample size requirement of the study was 196 for each group. We had a recruitment target of 200 patients with $4-5 \%$ patient's drop-out rate.

\section{Inclusion criteria}

- $\quad$ Age: 20-60 years

- Patients with abnormal symptoms like profuse white discharge, post coital bleeding, inter-menstrual bleeding or postmenopausal bleeding
- Patients with clinically unhealthy cervix diagnosed by speculum examination like cervical erosion, cervicovaginitis, cervical polyp, condylomas etc.

\section{Exclusion criteria}

- Patients with bleeding at the time of examination

- Women with frank invasive cancer

- Pregnant women.

The present study was carried out at Vydehi Institute of Medical Sciences and Research Centre, in the Department of Obstetrics and Gynaecology OPD. A total of 200 patients attending the Gynecology OPD were enrolled in the study. Patients to be screened were explained the procedure to be performed, written informed consent was taken and the relevant obstetrical and gynecological history was also taken, with the patient being reassured that the procedure was painless.

Firstly, a Pap smear was taken with Ayre's spatula and cytobrush and was evaluated by the Bethesda system. Following the Pap smear, VIA was performed with 5\% acetic acid followed by Lugol's iodine test (VILI). Results of VIA and VILI were recorded after waiting for 1 minute as negative or positive. All the tests were performed by trained residents and faculty who did not know the aims and objective of the study, and was also done separately by different residents/faculty.

All the patients in the study were subjected to colposcopy. The cervix was inspected with the naked eye than with a colposcope. After taking the Pap smear, the cervix was washed with normal saline and visualized, followed by application of 5\% acetic acid and visualization by colposcope followed by application of Lugol's iodine and colposcopy. Grading of lesion was done according to the Reid's colposcopic index. Biopsy was done for confirmation of lesion if either of the three screening tests or colposcopy had a positive finding or if the Pap smear reported ASCUS, for confirmation of lesion. Collected data was statistically analyzed to determine specificity and sensitivity, Positive Predictive Value (PPV), Negative Predictive Value (NPV) of Pap smear, VIA, VILI alone and Pap+VIA+VILI and VIA+VILI. The patients who reported normal on all screening tests and colposcopy were called for annual follow-up. Patients who were positive at either of the screening tests or colposcopy underwent biopsy and were treated according to the grade of the lesion (6-monthly follow-up and repeat Pap, loop electrode excision procedure (LEEP), hysterectomy, Wertheime's hysterectomy).

\section{RESULTS}

Author studied a total of 200 patients, median age of the patients was 39 years (range: 23-48 years) and median parity 3 (range: $0-5$ ). Most of the patients were in age category of $31-40$ years accounting for about $79 \%$ of the 
total patients. Among the study group 16 were para 1,68 were para 2, 76 were para 3 and 40 women were para 4 or more. Of the total patients studied, majority of women $(78.5 \%)$ were from upper lower and lower middle socioeconomic strata of society. Among 200 women studied, $53 \%$ were illiterate, $37 \%$ were just literate to middle and
$10 \%$ had secondary and above education. 156 patients (77.8\%) had single sexual partner, while $44(22.2 \%)$ had 2 or more sexual partners. Majority of the women were of lower socio economic status $154(78 \%)$ and from rural area $146(73 \%)$.

Table 1: Correlation between Pap smear and biopsy $(n=200)$.

\begin{tabular}{|c|c|c|c|c|c|c|c|}
\hline \multirow{2}{*}{ PAP Smear } & \multicolumn{7}{|l|}{ Biopsy } \\
\hline & Metaplasia & Cervicities & CIN1 & CIN2 & CIN3 & Invasive carcinoma & Total \\
\hline Normal & 3 & 110 & 21 & 8 & 0 & 4 & 146 \\
\hline Inflammatory & 0 & 11 & 9 & 2 & 0 & 0 & 22 \\
\hline ASCUS & 0 & 3 & 4 & 0 & 0 & 0 & 7 \\
\hline LSIL & 0 & 7 & 11 & 0 & 0 & 0 & 18 \\
\hline HSIL & 0 & 0 & 0 & 4 & 3 & 0 & 7 \\
\hline Malingnant cells & 0 & 0 & 0 & 0 & 0 & 0 & 0 \\
\hline Total $(\%)$ & $3(1.7)$ & $131(65.5)$ & $45(22.4)$ & $14(6.8)$ & $3(1.7)$ & $4(1.9)$ & 200 \\
\hline
\end{tabular}

32 cases out of 200 women were positive on Pap smear. 66 out of 200 women were positive on Biopsy. Pap smear was positive in 22 out of 66 biopsy proven positive cases.

Table 2: Evaluation of Colposcopy compared to cervical biopsy $(\mathbf{n}=\mathbf{2 0 0})$.

\begin{tabular}{|c|c|c|c|c|c|c|c|}
\hline \multirow{2}{*}{ Colposcopy } & \multicolumn{7}{|l|}{ Biopsy } \\
\hline & Metaplasia & Cervicities & CIN1 & CIN2 & CIN3 & Invasive carcinoma & Total \\
\hline Normal & 0 & 111 & 7 & 4 & 2 & 0 & 124 \\
\hline Erosion cervix & 1 & 7 & 3 & 1 & 0 & 0 & 12 \\
\hline Inflammatory changes & 1 & 2 & 6 & 3 & 0 & 0 & 12 \\
\hline Polyps & 0 & 1 & 9 & 4 & 1 & 0 & 15 \\
\hline Leucoplakia & 0 & 2 & 0 & 1 & 0 & 1 & 4 \\
\hline $\mathrm{AW}$ areas & 0 & 4 & 7 & 1 & 0 & 1 & 13 \\
\hline Punctate pattern & 1 & 1 & 7 & 0 & 0 & 1 & 10 \\
\hline Mosaic pattern & 0 & 1 & 1 & 0 & 0 & 0 & 2 \\
\hline Atypical vessels & 0 & 0 & 0 & 0 & 0 & 0 & - \\
\hline Unsatisfactory & 0 & 2 & 5 & 0 & 0 & 1 & 8 \\
\hline Total & $3(1.7)$ & $131(65.5)$ & $45(22.4)$ & $14(6.8)$ & $3(1.7)$ & $4(1.9)$ & 200 \\
\hline
\end{tabular}

77 cases out of 200 women were positive on Colposcopy. 66 out of 200 women were positive on Biopsy. Colposcopy was positive in 54 out of 66 biopsy proven positive cases.

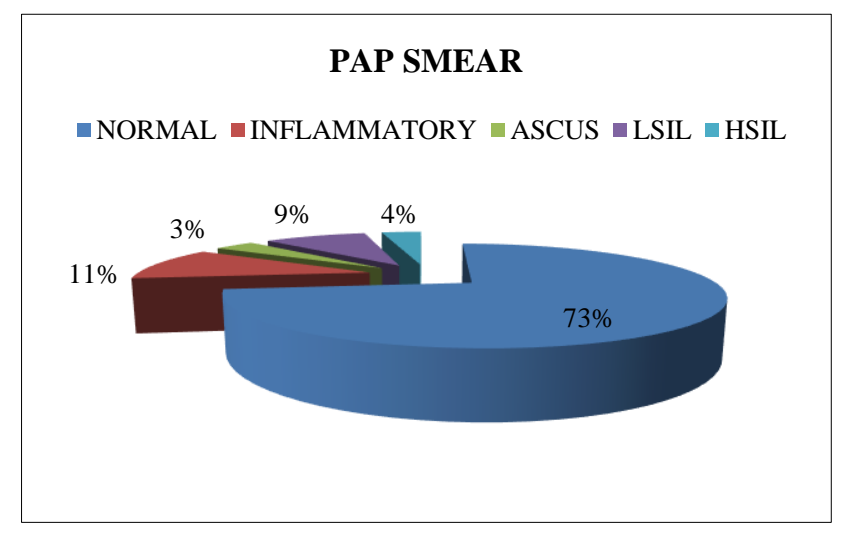

Figure 1: Pie diagram showing distribution of pap smear findings.

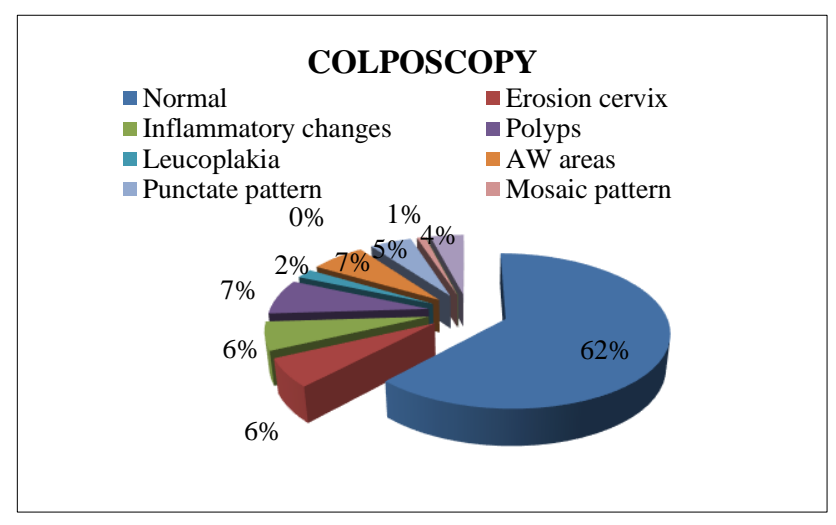

Figure 2: Distribution of colposcopy appearance of cervix. 
Only $5 \%$ of the women had the history of use of barrier method of contraception. 156 patients $(77.8 \%)$ had single sexual partner, while $44(22.2 \%)$ had 2 or more sexual partners. All the patients were married; mostly $(84.3 \%)$ in a monogamous setting. 186 clients $(92.9 \%)$ were Hindu, while the rest were Muslims. The commonest presenting complaint was vaginal discharge $(182 / 200 ; 91 \%$ of the patients). Other presenting complaints were postcoital bleeding (10/200), intermenstrual bleeding (2/200), postmenopausal bleeding $(4 / 200 ; 2 \%)$ and pruritis vulvae $(2 / 200 ; 1 \%)$.

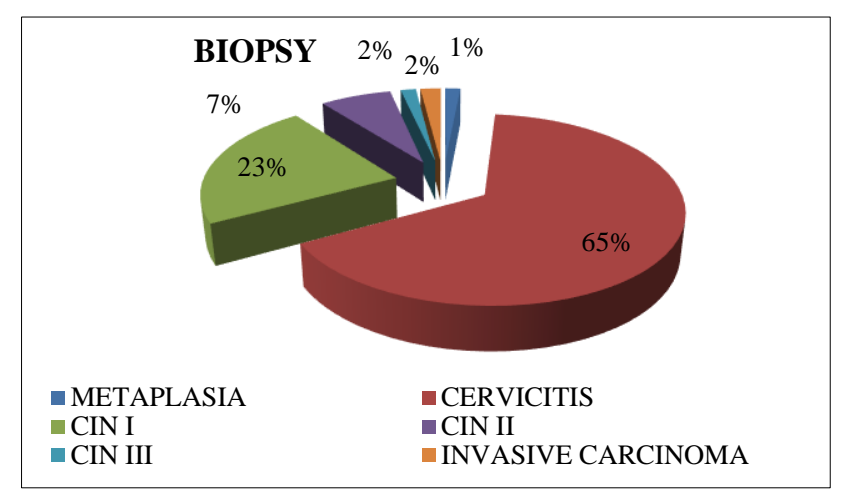

Figure 3: Distribution of Biopsy findings.

Table 3: Diagnostic efficacy of tests.

\begin{tabular}{|lll|}
\hline Efficacy of each test & $\begin{array}{l}\text { PAP } \\
\text { Smear }\end{array}$ & Colposcopy \\
\hline Sensitivity & $33.33 \%$ & $81.82 \%$ \\
\hline Specificity & $92.54 \%$ & $82.84 \%$ \\
\hline Positive predictive value & $68.75 \%$ & $70.13 \%$ \\
\hline Negative predictive value & $73.81 \%$ & $90.24 \%$ \\
\hline Percentage of false negatives & $66.67 \%$ & $18.18 \%$ \\
\hline Percentage of false positives & $7.4 \%$ & $17.16 \%$ \\
\hline Accuracy & $73 \%$ & $82.5 \%$ \\
\hline
\end{tabular}

PAP smear was taken for all patients. $73 \%$ of smear was found to be normal, $11 \%$ showed inflammatory atypia, 9\% showed LSIL, 3.5\% showed ASCUS and $3.5 \%$ showed HSIL (Figure 1). Among the 200 cases studied, $38 \%(76 / 200)$ were diagnosed as colposcopically abnormal. Among the abnormal cases, AW areas were diagnosed in $6.5 \%$. Punctate pattern of vessels was seen in $5 \%$ of women. Normal findings was present in $62 \%$, Erosion cervix in $6 \%$, inflammatory changes were seen in $6 \%$ and polyps were diagnosed in $7.5 \%$, lecoplakia was found in $2 \%$ and unsatisfactory colposcopy finding was seen in 4\% (Figure 2). All 200 cases were subjected to biopsy. Majority of cases, $65.5 \%$ cervicitis, $22.4 \%$ had CIN I, $6.8 \%$ had CIN II, $1.7 \%$ had CIN III, $1.9 \%$ had invasive carcinoma respectively (Figure 3). 32 cases out of 200 women were positive on Pap smear. 66 out of 200 women were positive on Biopsy. Pap smear was positive in 22 out of 66 biopsy proven positive cases (Table 1). 77 cases out of 200 women were positive on Colposcopy. 66 out of 200 women were positive on Biopsy. Colposcopy was positive in 54 out of 66 biopsy proven positive cases
(Table 2). Pap smear is found to have sensitivity of $33.33 \%$ and specificity of $92.54 \%$ and Colposcopy is found to have sensitivity of $81.82 \%$ and specificity of $82.84 \%$ (Table 3).

\section{DISCUSSION}

Cancer of the cervix is a leading cause of morbidity and mortality among women worldwide. Therefore, to curb the disease, there is a need to develop a screening test that has good sensitivity and specificity. Cervical cancer was the third most frequent cancer worldwide, in women after breast and colorectal carcinoma. However, invasive cancer of the cervix was considered to be a preventable condition as it is associated with a long pre invasive stage (CIN) making it amenable to screening and treatment. In the present study screening was done in 200 women with abnormal symptoms like excessive white discharge, post coital bleeding, postmenopausal bleeding etc., women with unhealthy cervix, and women with dysplastic smears, with colposcopy and its results were correlated with PAP smear and biopsy to determine the sensitivity and specificity of these methods in detecting CIN. We studied a total of 200 patients where median age of the patients was 39 years (range: 23-48 years) and median parity 3 (range: 0-5). Most of the patients were in age category of 31-40 years accounting for about $79 \%$ of the total patients. Among the study group 16 were para 1, 68 were para 2, 76 were para 3 and 40 women were para 4 or more. Of the total patients studied, majority of women $(78.5 \%)$ were from the upper lower and lower middle socio-economic strata of society. Majority of the studied patients were from rural areas. Among 200 women studied, $53 \%$ were illiterate, $37 \%$ had just literate to middle and $10 \%$ had secondary and above education. 156 patients $(77.8 \%)$ had single sexual partner while 44 (22.2\%) had multiple ( 2 or more) sexual partners. All the patients were married; mostly $(84.3 \%)$ in a monogamous setting. 186 clients $(92.9 \%)$ were Hindu, while the rest were Muslims. Only 5\% of the women had the history of use of barrier method of contraception. The commonest presenting complaint was vaginal discharge (182/200; $91 \%$ of the patients). Other presenting complaints were postcoital bleeding (10/200), intermenstrual (2/200), postmenopausal bleed $(4 / 200 ; 2 \%)$ and pruritus vulvae $(2 / 200 ; 1 \%)$. PAP smear was taken for all patients. $73 \%$ of smear was found to be normal, $11 \%$ showed inflammatory atypia, 9\% showed LSIL, 3.5\% showed ASCUS and $3.5 \%$ showed HSIL. Among the 200 cases studied, 38\% (76/200) were diagnosed as colposcopically abnormal. Among the abnormal cases, AW areas were diagnosed in $4 \%$. Punctate pattern of vessels was seen in $5 \%$ of women. Normal findings was present in $62 \%$, Erosion cervix in $6 \%$, inflammatory changes were seen in $6 \%$ and polyps were diagnosed in $7.5 \%$, leucoplakia was found in $2 \%$ and unsatisfactory colposcopy finding was seen in $14 \%$ and underwent endocervical cure. 32 cases out of 200 women were positive on Pap smear. 66 out of 200 women were positive on Biopsy. Pap smear was positive in 22 out of 66 biopsy proven positive cases. 
A prospective study was undertaken Londhe $\mathrm{M}$ et al, to determine the sensitivity and specificity of acetic application to the cervix followed by naked eye visualization as a screening test for detection of cervical intraepithelial neoplasia. ${ }^{7}$ Authors studied Three hundred and seventy two sexually active woman in the reproductive age group were studied. The reported lower sensitivity (13.2\%) may be explained by the fact that the author included women in reproductive age irrespective of the presence of clinical sign and symptoms which may have resulting in overrepresentation of healthy patients in the study. Presence of clinical sign and symptoms and unhealthy cervix on speculum examination have increased the representation of cases of carcinoma cervix in our study, thus increasing the specificity (33.33\%). The retrospective analysis by Shalini $\mathrm{R}$ et al, on cytologic, colposcopic and histopathologic evaluation on the cases of post-coital bleeding. ${ }^{8}$ The author reported a specificity of $56 \%$ which is far higher that what has been reported by our study. It can be explained by the fact that study included only the patients with complains of post coital bleeding, which is very common in patients with ca cervix, factor which could have led to over-representation of the ca cervix cases in the above mentioned study. The study by Basu PS et al, on visual inspection with acetic acid and cytology in the early detection of cervical neoplasia had similar inclusion criteria and patients profile and comparable specificity and sensitivity values $(29.5 \%) .{ }^{9}$ The study Pete I, et al on value of colposcopy in screening cervical carcinoma reported a sensitivity of $47 \%$ which was higher than our results. ${ }^{10}$ This can be explaining by the fact that the majority of the patients in this study were admitted for histological confirmation of atypical colposcopic and/or cytologic findings, which may have caused the over-representation of the carcinoma cervix cases. The specificity reported in various other studies were comparable (90-96.3\%).

Most of the reports quote the sensitivity of colposcopy between 87 - 99 percent, which is comparable to present. Olaniyan OB, did a meta-analysis of eight longitudinal studies and compared the correlation of colposcopy impression with biopsy results. ${ }^{11}$ Colposcopy sensitivity was found to be $87 \%$ which agreed exactly with histology in $61 \%$ of cases. In the present study the sensitivity colposcopy impression was found to be $81 \%$. Massadet al, reported a sensitivity of $89 \% .^{12}$ Specificity reported in various studied differed widely from $15 \%$ to $87 \%$ which may be explained by difference in the inclusion criteria adopted in various studies. Studies with similar inclusion criteria have reported comparable sensitivity valuesr.

\section{CONCLUSION}

In this study, pap smear was taken for all patients. $73 \%$ of smear was found to be normal, $11 \%$ showed inflammatory atypia, 9\% showed LSIL, 3.5\% showed ASCUS and 3.5\% showed HSIL. Among the 200 cases studied, $38 \%(76 / 200)$ were diagnosed as colposcopically abnormal. Among the abnormal cases, AW areas were diagnosed in $4 \%$. Punctate pattern of vessels was seen in $5 \%$ of women. Normal findings was present in $62 \%$, Erosion cervix in $6 \%$, inflammatory changes were seen in $6 \%$ and polyps were diagnosed in $7.5 \%$, lecoplakia was found in $2 \%$ and unsatisfactory colposcopy finding was seen in $14 \%$ and underwent endocervical cure. 32 cases out of 200 women were positive on Pap smear. 66 out of 200 women were positive on Biopsy. Pap smear was positive in 22 out of 66 biopsy proven positive cases.

The specificity reported in various other studies were comparable (90-96.3\%). Most of the reports quote the sensitivity of colposcopy between $87-99$ percent, which is comparable to present. Specificity reported in various studied differed widely from $15 \%$ to $87 \%$ which may be explained by difference in the inclusion criteria adopted in various studies. Studies with similar inclusion criteria have reported comparable sensitivity values

From our study we are of conclusion that Pap smear is found to have sensitivity of $33.33 \%$ and specificity of $92.54 \%$ and Colposcopy is found to have sensitivity of $81.82 \%$ and specificity of $82.84 \%$.

\section{Funding: No funding sources}

Conflict of interest: None declared

Ethical approval: The study was approved by the Institutional Ethics Committee

\section{REFERENCES}

1. Murray CJ, Vos T, Lozano R. Disability-adjusted life years (DALYs) for 291 diseases and injuries in 21 regions, 1990-2010: a systematic analysis for the global burden of disease study 2010. Lancet. 2012;380:2197-223.

2. Chisholm D, Abegunde D, Mendis S. Scaling up action against non-communicable diseases. How much it costs? WHO, 2011. Available at: www.who.int/nmh/publication/cost_of_inaction/en/. Accessed 27 May 2019.

3. Sankaranarayanan R, Nessa A, Esmy PO, Dangou JM. Visual inspection methods for cervical cancer prevention. Best Pract Res Clin Obstet Gynaecol. 2012;26:221-32.

4. Sankaranarayanan R, Nene BM, Shastri SS, Jayant $\mathrm{K}$, Muwonge R, et al. HPV screening for cervical cancer in rural India. N Engl J Med. 2009;360:138594.

5. Goldie SJ, Gaffikin L, Goldhaber-Fiebert JD, Gordillo-Tobar A, Levin C, et al. Cost-effectiveness of cervical-cancer screening in five developing countries. N Engl J Med. 2005;353:2158-68.

6. Forman D, de Martel C, Lacey CJ, Soerjomataram I, Lortet-Tieulent J, Bruni L et al. Vaccine. Global burden of human papillomavirus and related diseases. 2012;30(5):F12-23.

7. Londhe M, George SS, SeshadriL. Detection of CIN by naked eye visualization after application of acetic acid. Indian J Cancer. 1997;34(2):88-91. 
8. Shalini R, Amita S, Neera MA. How alarming is post-coital bleeding: a cytologic, colposcopic and histopathologic evaluation. Gynecol Obstet Invest. 1998;45(3):205-8.

9. Basu PS, Sankaranarayanan R, Mandal R, Roy C, Das P, Choudhury D, Bhattacharya D, et al. Calcutta cervical cancer early detection group. Visual inspection with acetic acid and cytology in the early detection of cervicalneoplasia in Kolkata, India. Int $\mathbf{J}$ Gynecol Cancer. 2003;13(5):626-32.

10. Pete I, Toth V, Bosze P. The value of colposcopy in screening cervical carcinoma. Eur J Gynecol Onco. 1998;19(2):120-2.
11. Olaniyan OB. Validity of colposcopy in the diagnosis of early cervical neoplasia a review - Af J Reprod Health. 2002;6:59-69.

12. Massad LS, Collins. 'Strength of Correlation between colposcopic impression and biopsy. J Gynaecol Oncol. 2003;89(3):424-8.

Cite this article as: Mohan $\mathrm{P}$, Lakshmidevi M, Venkatesh S. Comparative study of papanicolaou smear and colposcopy in the evaluation of cervical lesions. Int J Reprod Contracept Obstet Gynecol 2019;8:2400-5. 